\title{
The FERRUM project: Experimental lifetimes and transition probabilities from highly excited even $4 d$ levels in Fe II
}

\author{
H. Hartman ${ }^{1,2}$, H. Nilsson ${ }^{2}$, L. Engström ${ }^{3}$, and H. Lundberg ${ }^{3}$ \\ 1 Material Sciences and Applied Mathematics, Malmö University, 20506 Malmö, Sweden \\ e-mail: Henrik. Hartman@mah.se \\ 2 Lund Observatory, Lund University, Box 43, 22100 Lund, Sweden \\ 3 Department of Physics, Lund University, Box 118, 22100 Lund, Sweden
}

Received 23 January 2015 / Accepted 25 August 2015

\begin{abstract}
We report lifetime measurements of the 6 levels in the $3 \mathrm{~d}^{6}\left({ }^{5} \mathrm{D}\right) 4 \mathrm{~d} \mathrm{e}{ }^{6} \mathrm{G}$ term in Fe II at an energy of $10.4 \mathrm{eV}$, and $f$-values for 14 transitions from the investigated levels. The lifetimes were measured using time-resolved laser-induced fluorescence on ions in a laserproduced plasma. The high excitation energy, and the fact that the levels have the same parity as the the low-lying states directly populated in the plasma, necessitated the use of a two-photon excitation scheme. The probability for this process is greatly enhanced by the presence of the $3 \mathrm{~d}^{6}\left({ }^{5} \mathrm{D}\right) 4 \mathrm{p} \mathrm{z} \mathrm{z}^{6} \mathrm{~F}$ levels at roughly half the energy difference. The $f$-values are obtained by combining the experimental lifetimes with branching fractions derived using relative intensities from a hollow cathode discharge lamp recorded with a Fourier transform spectrometer. The data is important for benchmarking atomic calculations of astrophysically important quantities and useful for spectroscopy of hot stars.
\end{abstract}

Key words. atomic data - methods: laboratory: atomic - techniques: spectroscopic

\section{Introduction}

The present work reports on measurements of transition data involving high-excitation levels and lines of singly ionized iron, Fe II. The complex and line rich spectra of iron are observed in a wide variety of objects, such as stars, interstellar medium, solar coronae and quasars. In spectroscopy of stellar photospheres the atomic transitions most often appear as absorption lines. To use the spectral line for quantitative analysis of the population distribution and abundance determination in a stellar object, reliable $f$ values must be available.

To meet the demands for accurate atomic data, Johansson et al. (2002) initiated the FERRUM project with the goal of providing evaluated transition data for astrophysical applications. Transitions from levels with different excitation potentials, and with a range of transition probabilities have been measured and calculated for the iron group elements, see e.g. Sikström et al. (1999), Hartman et al. (2005), Gurell et al. (2010), and references therein. The present paper extends the data of highly excited levels, around $10 \mathrm{eV}$. The amount of data available from experimental studies is limited, and the majority must be provided by calculations. The experimental data is important to benchmark different calculations and to provide the user with reliable uncertainties and priorities between data sets. This is especially true for the complex ions where level mixing is important and hard to predict.

A common technique to derive the oscillator strength is to combine measurements of the radiative lifetime of an upper level with relative intensities for all decay channels. The present paper reports on values using this approach, where the radiative lifetimes are determined with Time-Resolved Laser Induced Fluorescence (TR-LIF) from ions produced in a laser ablation plasma. These are combined with branching fractions obtained from intensity calibrated Fourier transform spectra using a hollow cathode discharge as a light source.

\section{Radiative lifetime measurements}

Figure 1 presents a partial energy level diagram for Fe II showing the large number of parent terms. The close similarity in the energy differences between $4 \mathrm{~s}$ and $4 \mathrm{p}$ for all parent terms (the promotion energy), give rise to a high Fe II line density in the region around $230-260 \mathrm{~nm}$. In addition, the $4 \mathrm{~s}-4 \mathrm{p}$ and $4 \mathrm{p}-5 \mathrm{~s} / 4 \mathrm{~d}$ transition arrays have similar energy differences, and the latter transitions thus also appear in the same wavelength region. The target of the present study is the $3 d^{6}\left({ }^{5} \mathrm{D}\right) 4 \mathrm{~d} \mathrm{e}^{6} \mathrm{G}$ term. This term has even parity which is the same as the ground configuration $3 d^{6}\left({ }^{5} \mathrm{D}\right) 4 \mathrm{~s}$. This, and the large energy difference makes ${ }^{6} \mathrm{G}$ impossible to excite in a one-photon process. However, the use of an intense laser beam opens the possibility for two-photon excitations from the ground term, which has a high relative population in the ablation plasma.

The TR-LIF set-up at Lund High Power Laser Facility is recently described in detail by Engström et al. (2014), where a study similar to this, but for Cr II, is reported; here we only emphasize the important aspects. The set-up contains two Nd:YAG lasers operating at $10 \mathrm{~Hz}$. One of them (Continuum Surelite) is frequency doubled and focussed onto a rotating iron target placed inside a vacuum chamber to generate the ablation plasma. The second laser is an injection seeded and Q-switched Continuum NY-82. The $532 \mathrm{~nm}$ output from this laser was temporally compressed using stimulated Brillouin scattering in water before pumping a dye laser (Continuum Nd-60) using DCM ${ }^{1}$.

\footnotetext{
1 4-(Dicyanomethylene)-2-methyl-6-(4-dimethylaminostyryl)-4Hpyran.
} 


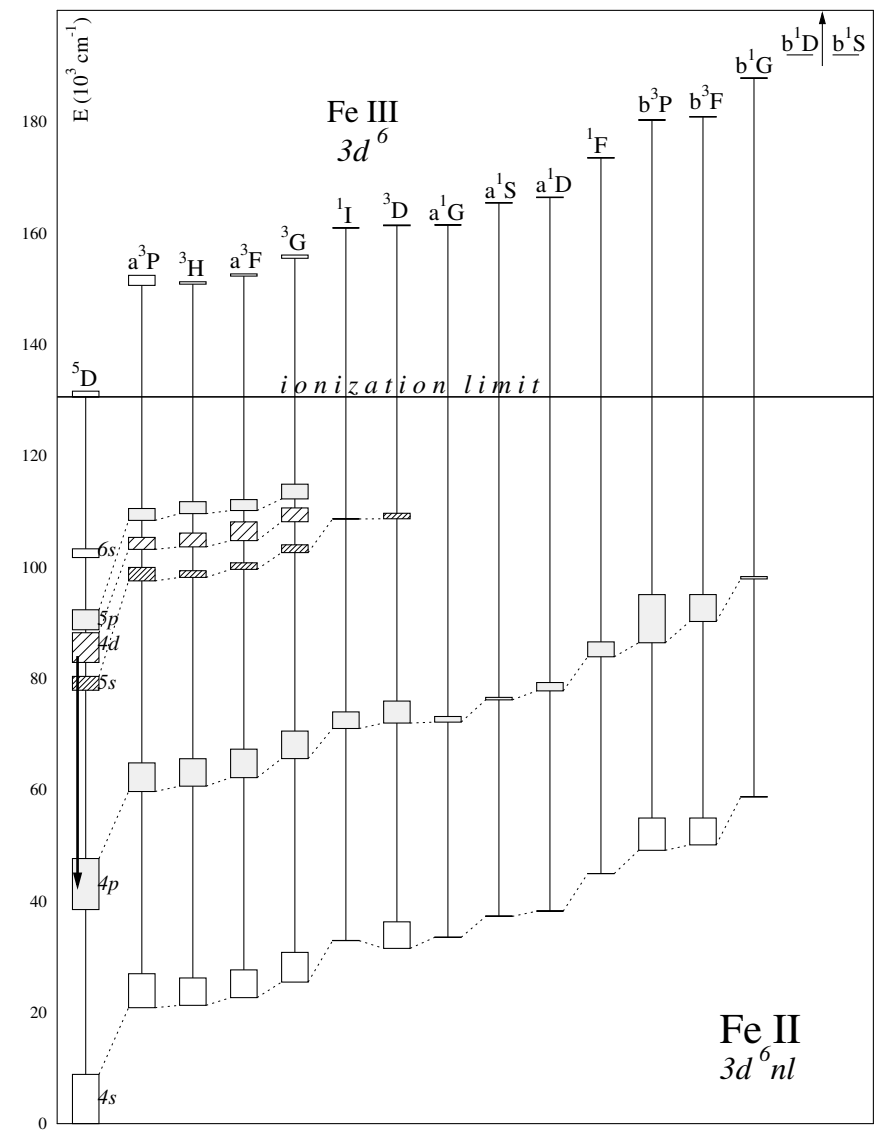

Fig. 1. A partial level diagram of Fe II showing the parent term structure, and the similar energies for all $4 s-4 p$ and $4 p-5 s / 4 d$ transitions. The arrow marks the transitions investigated in this study.

To obtain the $237 \mathrm{~nm}$ radiation needed for the two-photon excitation, the output was frequency tripled in $\mathrm{KH}_{2} \mathrm{PO}_{4}$ (KDP) and $\mathrm{BaB}_{2} \mathrm{O}_{4}$ (BBO) crystals and Raman shifted in a high pressure hydrogen cell. The laser-induced fluorescence was detected by a $1 / 8 \mathrm{~m}$ monochromator with a $280 \mu \mathrm{m}$ wide entrance slit oriented parallel to the excitation laser beam and perpendicular to the ablation laser. All measurements were performed in the second spectral order giving an instrumental line width of $0.5 \mathrm{~nm}$. The fluorescence signal was recorded with a micro-channel plate photomultiplier (PM) tube (Hamamatsu R3809U) with a rise time of $0.2 \mathrm{~ns}$, and digitized by a Tektronix oscilloscope with $2.5 \mathrm{GHz}$ analogue bandwidth. A second channel on the oscilloscope sampled the excitation laser pulse shape from a fast diode fed by scattered light from an attenuating filter. Each decay curve, as well as the temporal shape of the excitation pulse, was averaged over 1000 laser shots. The lifetimes were extracted using the program DECFIT (Palmeri et al. 2008). The program derives the lifetime from the measured laser pulse and the recorded decay. In the present experiment, the fluorescence data was fitted with a single exponential convoluted with the square of the measured laser pulse (since the upper state is excited through a two-photon absorption) and a constant background. The laser pulse had a temporal width (FWHM) of 2 ns. A typical example is shown in Fig. 2.

The final lifetimes given in Table 1 are the averages of around 20 measurements, performed during different days. The quoted uncertainties, between 10 and $17 \%$, include the statistical contributions from the fitting process as well as variations in

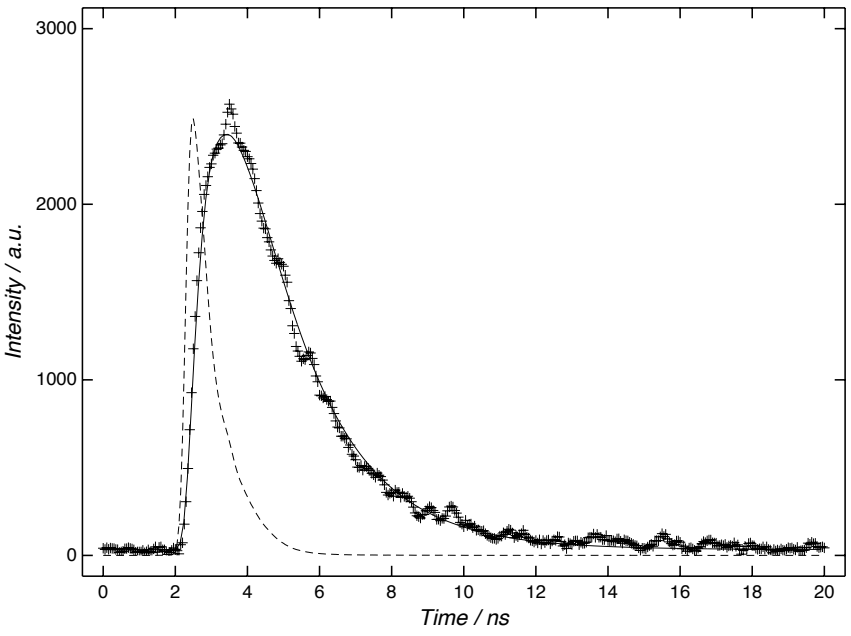

Fig. 2. The first $20 \mathrm{~ns}$ of the decay of the $3 \mathrm{~d}^{6} 4 \mathrm{de}^{6} \mathrm{G}_{11 / 2}$ level in Fe II following two-photon excitation from the ground state $\left(3 \mathrm{~d}^{6} 4 \mathrm{~s} \mathrm{a}^{6} \mathrm{D}_{9 / 2}\right)$. The evaluated lifetime is $2.0 \pm 0.2 \mathrm{~ns}$. Background subtracted data points $(+)$ are plotted together with a fitted single exponential decay convoluted by the square of the measured laser pulse (solid line). The dashed curve shows the squared laser pulse.

the results between the different measurements. The high probability of spectral coincidences between the two-photon pumping channel and a single photon excitation of the $4 p$ levels had to be thoroughly considered. Another consequence of the similarity between the $4 s-4 p$ and $4 p-4 d$ wavelengths is that the secondary cascades $(4 s-4 p)$ coincide with the measured primary decay $(4 p-4 d)$. The chosen pump and detection channels are given in Table1, and the most difficult cases will be discussed in detail below. All measurements are checked for blending lines, both in the excitation and decay channels, and three of the levels are discussed in more detail below.

The high $J$ value of the $\mathrm{e}^{6} \mathrm{G}_{13 / 2}$ level, and the $\Delta J=0, \pm 1$ restriction for E1 transitions, effectively limits the decay channels to only the $\left({ }^{5} \mathrm{D}\right) 4 \mathrm{p}$ level with $J=11 / 2, \mathrm{z}^{6} \mathrm{~F}_{11 / 2}$. Transitions to $4 \mathrm{p}$ levels belonging to other parent terms are several orders of magnitudes weaker. The pumping transition is located at $238.0 \mathrm{~nm}$, and the primary and secondary decays fall at $237.64 \mathrm{~nm}$ and $238.28 \mathrm{~nm}$, respectively. In this case the detection had to be shifted towards shorter wavelengths to avoid the influence from scattered laser light and secondary decay. However, shifting the detection wavelength too much to the blue brings in the fluorescence at $234.8 \mathrm{~nm}$ from $4 \mathrm{p} \mathrm{z}^{4} \mathrm{D}_{7 / 2}$ with a lifetime of $3.02 \mathrm{~ns}$ (Guo et al. 1992), which is excited by a single $238.0 \mathrm{~nm}$ photon. This effect was clearly observed in the decay curve as a weak multi-exponential shape which resulted in a longer lifetime in our single exponential analysis compared to detection at longer wavelengths. By stepping the detection wavelength from this decay towards the primary decay at $237.64 \mathrm{~nm}$, the contribution from $\mathrm{z}^{4} \mathrm{D}_{7 / 2}$ could be monitored, both in the derived lifetime and the fitted curve. From this behaviour and the known instrumental line width of $0.5 \mathrm{~nm}$ we estimated the maximum contribution from other decays to be $10 \%$. This is reflected in the larger total uncertainty quoted for this level, 14\%, compared to $11 \%$ for the other levels.

The level $\mathrm{e}^{6} \mathrm{G}_{11 / 2}$ was pumped at $237.2 \mathrm{~nm}$ from the ground state $a^{6} D_{9 / 2}$. The primary decay is at $237.0 \mathrm{~nm}$ whereas the secondary decay, $\mathrm{a}^{6} \mathrm{D}-\mathrm{z}^{6} \mathrm{~F}$, falls at $238.2 \mathrm{~nm}$. The monochromator was tuned to the blue side of the decay channel to minimize 
H. Hartman et al.: Lifetimes and $f$-values for 4 d-levels in Fe II

Table 1. Excitation and detection scheme for the measured levels, and results from this work and comparisons with previous calculations.

\begin{tabular}{llllllll}
\hline \hline Level & $E / \mathrm{cm}^{-1}$ & \multicolumn{2}{l}{ Pump channel } & \multicolumn{2}{l}{$\begin{array}{l}\text { Detection channel } \\
\text { level }\end{array}$} & $\lambda / \mathrm{nm}$ & \multicolumn{2}{l}{$\begin{array}{l}\text { Lifetime/ns } \\
\text { this work }\end{array}$} & ${\mathrm{K} 13^{a}}^{a}$ & $\mathrm{RU}^{b} 8^{b}$ \\
\hline $\mathrm{e}^{6} \mathrm{G}_{13 / 2}$ & 84035 & $\mathrm{a}^{6} \mathrm{D}_{9 / 2}$ & 237.99 & 237 & $2.1 \pm 0.3$ & 1.48 & 1.57 \\
$\mathrm{e}^{6} \mathrm{G}_{11 / 2}$ & 84296 & $\mathrm{a}^{6} \mathrm{D}_{9 / 2}$ & 237.26 & $236-237$ & $2.0 \pm 0.2$ & 1.46 & 1.56 \\
$\mathrm{e}^{6} \mathrm{G}_{9 / 2}$ & 84527 & $\mathrm{a}^{6} \mathrm{D}_{7 / 2}$ & 237.69 & $235-238$ & $1.9 \pm 0.2$ & 1.45 & 1.55 \\
$\mathrm{e}^{6} \mathrm{G}_{7 / 2}$ & 84710 & $\mathrm{a}^{6} \mathrm{D}_{3 / 2,7 / 2}$ & $237.10 ; 238.45$ & $235-263$ & $1.8 \pm 0.2$ & 1.55 & 1.55 \\
$\mathrm{e}^{6} \mathrm{G}_{5 / 2}$ & 84844 & $\mathrm{a}^{6} \mathrm{D}_{5 / 2}$ & 237.52 & $235-266$ & $1.8 \pm 0.2$ & 1.44 & 1.54 \\
$\mathrm{e}^{6} \mathrm{G}_{3 / 2}$ & 84938 & $\mathrm{a}^{6} \mathrm{D}_{1 / 2}$ & 238.21 & $235-236$ & $1.7 \pm 0.3$ & 1.43 & 1.53 \\
\hline
\end{tabular}

References. ${ }^{(a)}$ Kurucz (2013); ${ }^{(b)}$ Raassen \& Uylings (1998a,b).

scattered laser light in the detection. In addition, the contribution from secondary decays was avoided.

The level $\mathrm{e}^{6} \mathrm{G}_{7 / 2}$ can be populated by two-photon excitation from the lower levels a ${ }^{6} \mathrm{D}_{3 / 2,5 / 2,7 / 2}$. The excitation from $J=3 / 2$ was the best channel. The transitions from $J=7 / 2$ is close to the excitation route to $e^{6} \mathrm{G}_{13 / 2}$, but still possible. The transition from $J=5 / 2$ is close to the laser frequency and could not be used.

\section{Branching fractions}

The $B F$ for a transition from an upper level $i$ to a lower level $k$ can be expressed as:

$$
B F_{i k}=\frac{A_{i k}}{\sum_{j=1}^{N} A_{i j}}=\frac{I_{i k}}{\sum_{j=1}^{N} I_{i j}}
$$

Here $N$ is the total number of transitions from level $i$ and $j$ is summed over all lower levels. The first equality is the definition of $B F_{i k}$, i.e. the fraction of the atoms in level $i$ that decay through the channel $i k$. The second equality assumes the case of an optically thin plasma, where the observed photon intensity of a line, $I_{i j}$ measured as photons per second, is proportional to $A_{i j}$ (Thorne et al. 1999). When all intensities are measured on a common calibrated scale, $B F_{i k}$ can be derived from the measured intensities.

The lifetime of the upper level, $\tau_{i}$, puts the relative intensities on an absolute scale. With

$$
\tau_{i}=\frac{1}{\sum_{j=1}^{N} A_{i j}}
$$

and Eq. (1) we derive $A$ from

$A_{i k}=\frac{B F_{i k}}{\tau_{i}}$

This requires all lines from the upper level to be measured. In practice it is usually impossible to include all transitions, either because they occur outside the detector range or they are too weak to be observed. Thus, we rewrite Eq. (1) as:

$$
\begin{aligned}
B F_{i k} & =\frac{I_{i k}}{\sum_{j=1}^{N} I_{i j}}=\frac{1}{1+\sum_{j=1, j \neq k}^{n} \frac{I_{i j}}{I_{i k}}+\sum_{j=n+1}^{N} \frac{I_{i j}}{I_{i k}}} \\
& \approx \frac{1}{1+\frac{1}{I_{i k}} \sum_{j=1, j \neq k}^{n} I_{i j}+\frac{1}{A_{i k}} \sum_{j=n+1}^{N} A_{i j}} .
\end{aligned}
$$

Here $n$ is the number of observed lines and the sum over the intensity ratios for the unobserved lines is approximated using the theoretical transition probabilities from Kurucz (2013).

The iron plasma was produced in a hollow cathode discharge (HCD), operated with neon as carrier gas and using currents between 0.6 and $0.8 \mathrm{~A}$ and pressures between 1 and 2 torr. The light emitted was recorded with a Fourier transform spectrometer (Chelsea Instruments FT500) in the range 27 640-52 $290 \mathrm{~cm}^{-1}$ and detected by a solar blind PM tube (Hamamatsu R166). In total, five spectra were measured. While a higher current in the HCD produced a more intense spectrum it also led to slightly wider lines due to the temperature dependent Doppler effect. Line widths between 0.18 and $0.25 \mathrm{~cm}^{-1}$ were observed with most of the spectra having $0.21 \mathrm{~cm}^{-1}$. To calibrate the intensity scale, three spectra with lower resolution were recorded using a deuterium lamp with known relative spectral radiance, measured at the Physicalisch-Technische Bundesanstalt, Berlin, Germany.

Despite the high spectral resolution of the spectrometer (resolving power about $2 \times 10^{5}$ ) the very line rich spectrum of $\mathrm{Fe}^{+}$ in this region produced a number of blends that must be taken into account to derive accurate experimental intensities. The first case is the $\mathrm{z}^{6} \mathrm{~F}_{11 / 2}-\mathrm{e}^{6} \mathrm{G}_{11 / 2}$ channel at $42328.78 \mathrm{~cm}^{-1}$, where there are two additional Fe II lines at 0.185 and $0.352 \mathrm{~cm}^{-1}$ higher wavenumbers, respectively (Johansson 1978; Nave \& Johansson 2013). This blending was handled by fitting a sum of three Gaussian shaped line components, constrained to have the same FWHM and their separations fixed to the accurately known values, using the computer code GFit (Engström 2014). One of these fits is shown in Fig. 3. Consistent line widths and relative intensities were obtained in all five measurements.

A more severe blending is found in the $\mathrm{z}^{6} \mathrm{~F}_{5 / 2}-\mathrm{e}^{6} \mathrm{G}_{7 / 2}$ channel at $42375.86 \mathrm{~cm}^{-1}$ where another Fe II line is only $0.11 \mathrm{~cm}^{-1}$ higher. Furthermore, contrary to the previous case the blending line is also significantly more intense. The experimental spectra were analysed using the same technique of constrained fitting as before. Also, in this case, we obtained the same FWHM as for resolved lines but the relative intensities of the two components varied somewhat between the five measurements. In addition to the three lines included in our work, Kurucz (2013) predicts an $A$ value larger than $10^{7} \mathrm{~s}^{-1}$ for four additional transitions $\left(41472,40478,40263\right.$ and $39925 \mathrm{~cm}^{-1}$ ) from the $\mathrm{e}^{6} \mathrm{G}_{7 / 2}$. Unfortunately all these lines are too severely blended in our spectra to be included in our analysis. This causes the so-called residual in Table 2, i.e. the sum of all theoretical $B F$ s from Kurucz (2013) for the unobserved lines from this level, to be as high as $34 \%$. Since the experimental $B F \mathrm{~s}$, according to Eq. (3), depends on this residual, our values for the $e^{6} G_{7 / 2}$ level must be considered less reliable than for the other levels which is reflected in the higher uncertainties. 


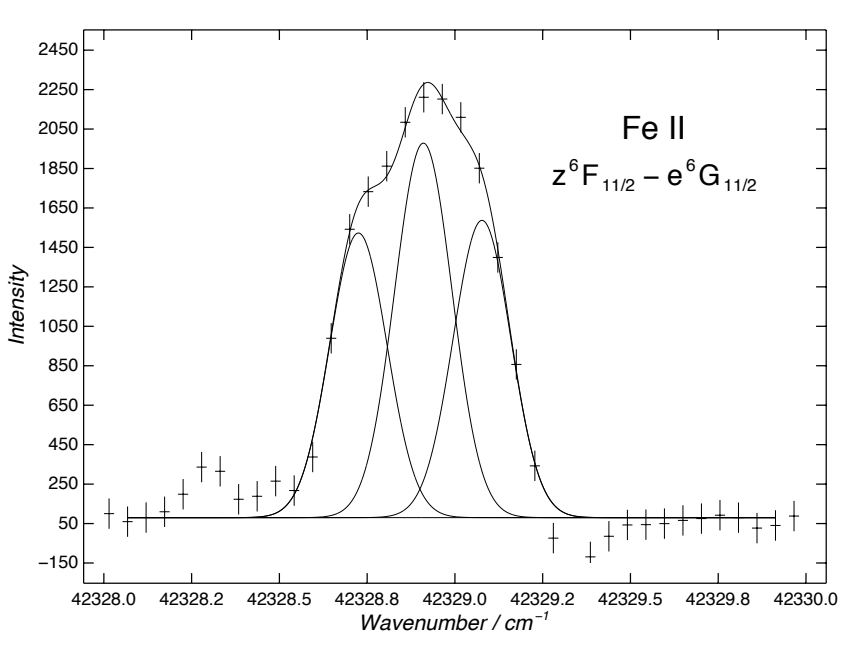

Fig. 3. The $4 \mathrm{p} \mathrm{z}^{6} \mathrm{~F}_{11 / 2}-4 \mathrm{~d} \mathrm{e}^{6} \mathrm{G}_{11 / 2}$ line at $42328.78 \mathrm{~cm}^{-1}$ blended by two other Fe II lines at 0.185 and $0.352 \mathrm{~cm}^{-1}$ higher wavenumber, respectively. These separations have been kept fixed in the fitting of the three Gaussian shaped lines.

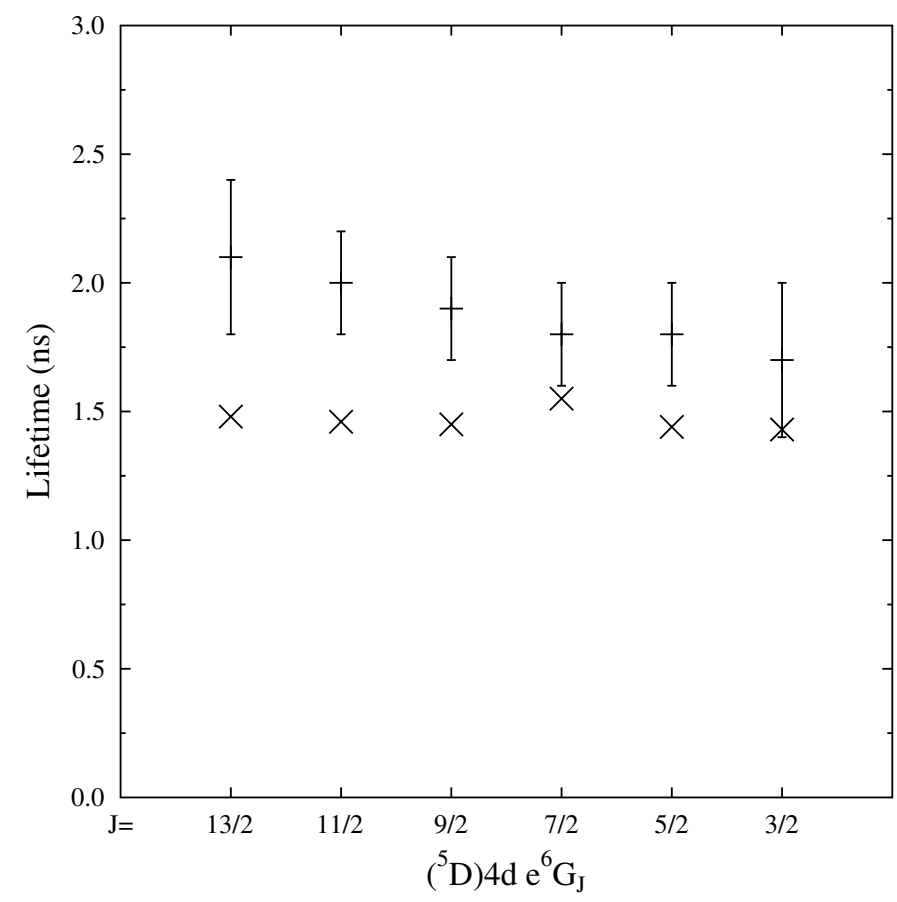

Fig. 4. Experimental lifetimes are plotted with error bars (+), whereas the theoretical lifetimes (Kurucz 2013) are marked with crosses $(\times)$.

The last example of blending is the close coincidence between $\mathrm{z}^{6} \mathrm{~F}_{7 / 2}-\mathrm{e}^{6} \mathrm{G}_{5 / 2}$ at $42607.80 \mathrm{~cm}^{-1}$ and a Fe II transition at $42607.79 \mathrm{~cm}^{-1}$. In addition, the lines can be expected to have similar intensities. Thus the 7/2-5/2 combination are omitted from the $B F$ analysis.

We present the final average $B F$ s and transition probabilities obtained in Table 2, where we include the theoretical results by Kurucz (2013) for comparison. The error estimate in the BFs includes the uncertainty in the intensity calibration, in the area determination of the peaks, and a 50\% uncertainty in the contribution from the unobserved branches obtained from Kurucz (2013), as described in Eq. (3). Since all but one of the observed decay branches are within $735 \mathrm{~cm}^{-1}$ of each other and, in addition, occur in a wavenumber region where the deuterium lamp calibration spectrum is quite flat, the uncertainty contribution from the calibration is small, about 3\%. However, in the present case the total uncertainty of the transition probabilities for the strong lines is dominated by the contribution from the lifetime measurements. For the weak lines, the uncertainty in the $B F$ is the dominating source. A more general discussion of uncertainties in experimental transition probabilities can be found in Sikström et al. (2002).

\section{Discussion}

Figure 5 shows a Hubble Space Telescope spectrum of the binary system chi Lupi (HD 141556), which has spectral types B9.5p HgMn and A2 Vm with effective temperatures of $T_{\text {eff }}=$ $10650 \mathrm{~K}$ and $9200 \mathrm{~K}$, respectively (Wahlgren et al. 1994). The investigated transitions $4 \mathrm{p}-4 \mathrm{~d} \mathrm{e}^{6} \mathrm{G}$ are observed as prominent absorption features around $235 \mathrm{~nm}$.

In Table 1 we compare our experimental lifetimes with the available theoretical data from Kurucz (2013) and Raassen \& Uylings (1998a). Kurucz (2013) applied a superposition of configurations method using a modified version of the Cowan codes (Cowan 1981), whereas Raassen \& Uylings (1998b) applied the orthogonal operator formalism. Both calculations make use of experimental level energies to improve the results. As seen in Table 1, the two theoretical results are within $7 \%$, but they are about $25 \%$ shorter and do not overlap with the experimental values, even taking the rather large uncertainties into account. A graphical comparison is shown in Fig. 4, where a weak tendency of lower experimental lifetimes for lower $J$ values is seen. This trend is within the error bars.

The discrepancy between measured and calculated $B F \mathrm{~s}$ is relatively small for most lines. An interesting exception are the transitions from $e^{6} G_{7 / 2}$, where there is a large difference between the two theoretical studies. Although both give similar lifetimes they differ significantly in the transition probabilities for individual lines. For two of the transitions our values support those of Kurucz (2013), whereas the $\mathrm{z}^{4} \mathrm{P}_{5 / 2}-\mathrm{e}^{6} \mathrm{G}_{7 / 2}$ line at $37743 \mathrm{~cm}^{-1}$, which is predicted to be quite intense is barely observable in our spectra. In addition, the large residual for this level, as discussed above, leads to a large uncertainty in the experimental $A$ values. The fact that the values by Kurucz (2013) differ significantly from his earlier 2010 calculations indicate theoretical difficulties for this level ${ }^{2}$. However, for all levels the resulting experimental $A$ values are a combination of lifetimes and $B F \mathrm{~s}$, and the discrepancy between the experimental and theoretical results is in most cases a consequence of the lifetime differences.

The general agreement, except for the mentioned $e^{6} G_{7 / 2}$, of the experimental $f$ values with the calculations by Kurucz (2013) and Raassen \& Uylings (1998a) provides us with confidence to use either set for abundance determinations and also for high-excitation lines represented by the transitions and levels investigated in this work. We have a preference for the calculations by Kurucz (2013) when compared with the experimental values, however, the discrepancy between experiment and theory has to be considered when estimating the uncertainty in the abundance determination or evaluation of the stellar atmosphere models. We recommend that the experimental data is used where available, and complemented with theoretical calculations for the other lines.

2 The calculations from 2010 (referenced as K10 in Kurucz notation) are not available online any longer, but replaced by the new data Kurucz (2013). 
H. Hartman et al.: Lifetimes and $f$-values for $4 \mathrm{~d}$-levels in Fe II

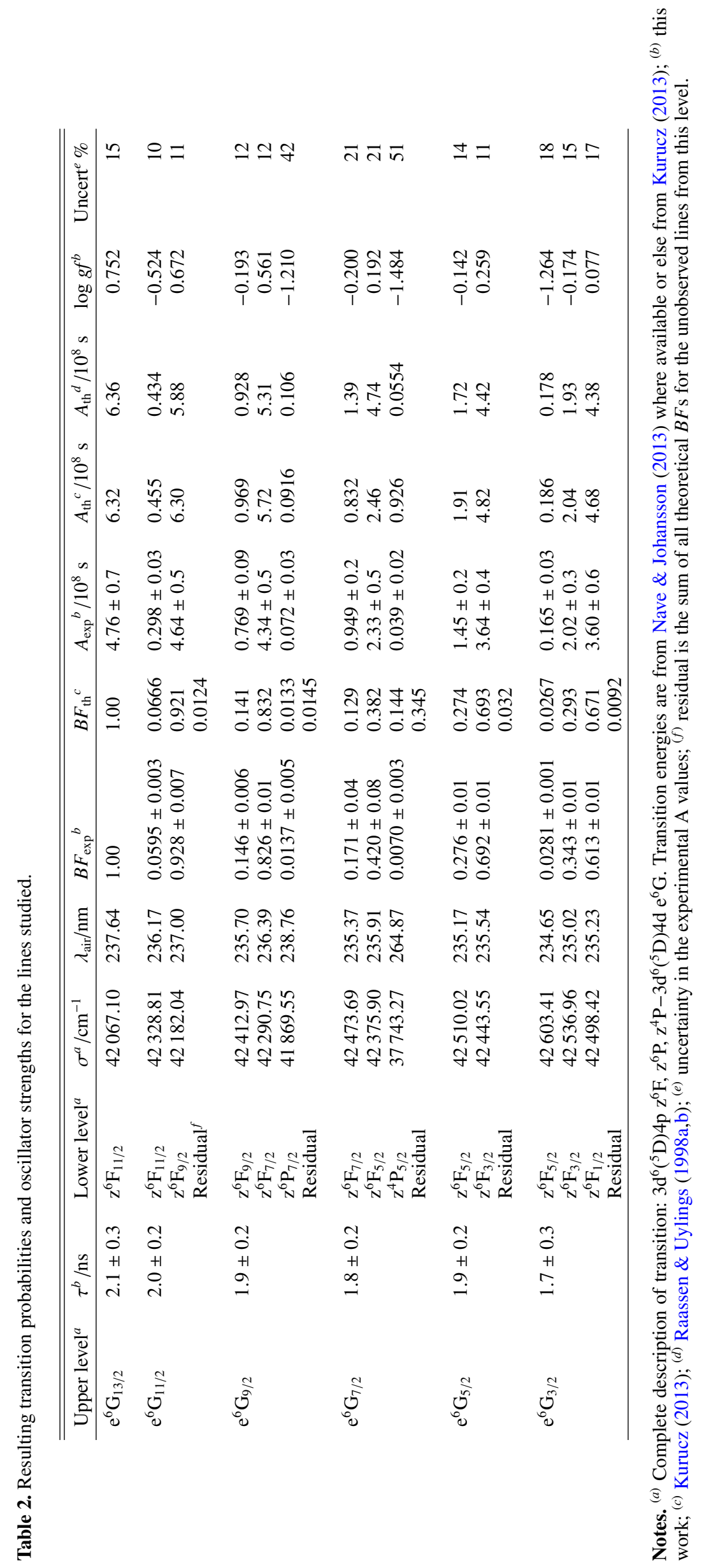



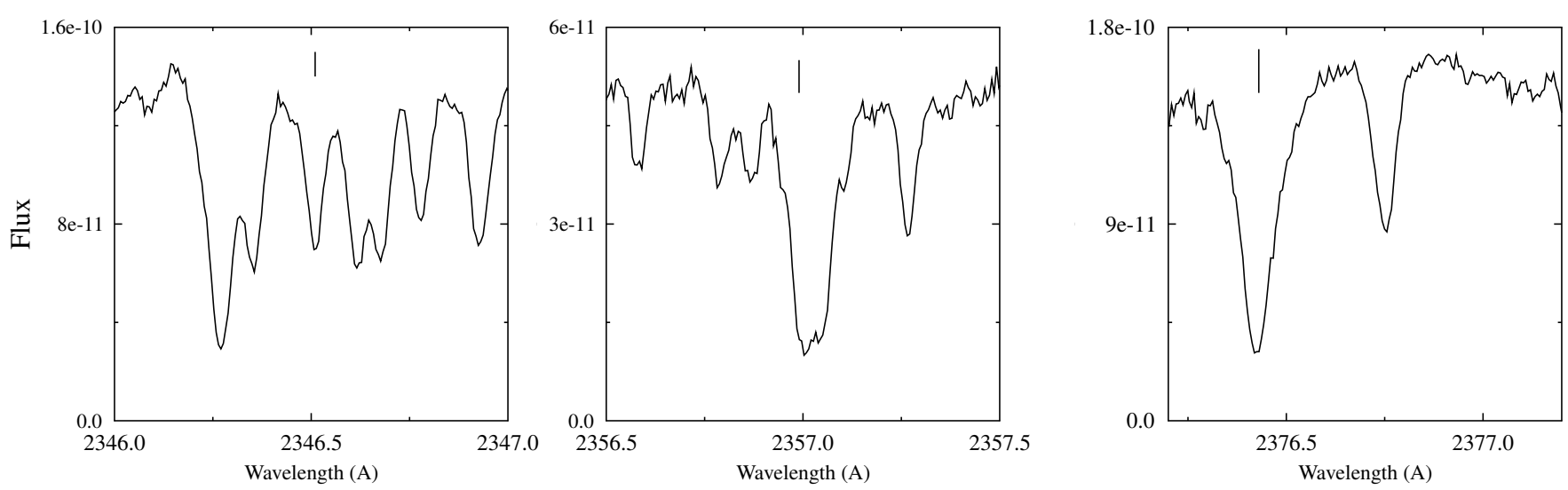

Fig. 5. Partial absorption spectrum of the chemically peculiar star chi Lupi, with the investigated Fe II lines marked with tickmarks. The spectrum is observed by the Hubble Space Telescope and its Goddard High Resolution Spectrograph, and is obtained from the MAST data archive at the Space Telescope Science Institute. See Brandt et al. (1999) for a complete identification list and a comparison with a synthetic spectrum.

Acknowledgements. We are grateful to the anonymous referee for valuable comments significantly improving the manuscript. This work was supported by the Swedish Research Council through the Linnaeus grant to the Lund Laser Centre and the Knut and Alice Wallenberg Foundation. H.H. gratefully acknowledges the grant No. 621-2011-4206 from the Swedish Research Council and support from the The Gyllenstierna Krapperup's Foundation. Figure 5 is based on observations made with the NASA/ESA Hubble Space Telescope, obtained from the MAST data archive at the Space Telescope Science Institute. STScI is operated by the Association of Universities for Research in Astronomy, Inc. under NASA contract NAS 5-26555.

\section{References}

Brandt, J. C., Heap, S. R., Beaver, E. A., et al. 1999, AJ, 117, 1505 Cowan, R. 1981, The Theory of Atomic Structure and Spectra (California: University of California Press, Berkeley)

Engström, L. 2014, GFit, http://kurslab-atom.fysik.1th.se/Lars/ GFit/Html/index.html

Engström, L., Lundberg, H., Nilsson, H., Hartman, H., \& Bäckström, E. 2014, A\&A, 570, A34
Guo, B., Ansbacher, W., Pinnington, E. H., Ji, Q., \& Berends, R. W. 1992, Phys. Rev. A, 46, 641

Gurell, J., Nilsson, H., Engström, L., et al. 2010, A\&A, 511, A68

Hartman, H., Schef, P., Lundin, P., et al. 2005, MNRAS, 361, 206

Johansson, S. 1978, Phys. Scr., 18, 217

Johansson, S., Derkatch, A., Donnelly, M. P., et al. 2002, Phys. Scr. T, 100, 71

Kurucz, R. 2013, http://kurucz.harvard.edu/atoms.html, accessed: 2015-03-03

Nave, G., \& Johansson, S. 2013, ApJS, 204, 1

Palmeri, P., Quinet, P., Fivet, V., et al. 2008, Phys. Scr., 78, 015304

Raassen, A. J. J., \& Uylings, P. H. M. 1998a, http://www. science.uva.nl/ pub/orth/iron/FeII.E1, accessed: 2006-02-14

Raassen, A. J. J., \& Uylings, P. H. M. 1998b, A\&A, 340, 300

Sikström, C. M., Schultz-Johanning, M., Kock, M., et al. 1999, J. Phys. B Atom. Mol. Phys., 32, 5687

Sikström, C. M., Nilsson, H., Litzen, U., Blom, A., \& Lundberg, H. 2002, J. Quant. Spectry. Radiat. Transf., 74, 355

Thorne, A., Litzén, U., \& Johansson, S. 1999, Spectrophysics: principles and applications (Springer Verlag)

Wahlgren, G. M., Adelman, S. J., \& Robinson, R. D. 1994, ApJ, 434, 349 